\title{
Bosonization theory for tunneling spectra in smooth edges of Quantum Hall systems
}

\author{
Sergio Conti ${ }^{\dagger}$ and Giovanni Vignale\# \\ † Scuola Normale Superiore, 56126 Pisa, Italy \\ \# Department of Physics, University of Missouri, Columbia, Missouri 65211, USA
}

(December 5, 2017)

\begin{abstract}
We calculate the spectral function of a smooth edge of a quantum Hall system in the lowest Landau level by means of a bosonization technique. We obtain a general relationship between the one electron spectral function and the dynamical structure factor. The resulting $I-V$ characteristics exhibit, at low voltage and temperature, power law scaling, generally different from the one predicted by the chiral Luttinger liquid theory, and in good agreement with recent experimental results.
\end{abstract}

Edges of Quantum Hall systems have been studied mainly as realizations of exotic correlated states of electrons. For example a sharp edge of a fractional quantum Hall fluid is described as a Chiral Luttinger Liquid (CLL) [1]. The main characteristic of a CLL is the lack of single particle excitations at the Fermi level: the elementary excitations are collective waves. Experimental data [2] seem to support this picture at filling factor $1 / 3$.

In many experiments the potential confining the electrons is slowly varying on the scale of the magnetic length $l=(\hbar c / e B)^{1 / 2}$. Under these conditions, the edge reconstructs itself [3]. For sufficiently smooth confining potential the density profile can be determined (on a coarse grained scale $\gtrsim l$ ) by the minimization of a local energy functional [4]. This leads to a picture of the edge as a broad compressible region characterized by fractional, non-uniform occupation of degenerate orbitals at the Fermi level. The single particle description is meaningless for such a state, and a full many-body treatment of the excitations is needed. As a first step in that direction, in a recent paper [5] we have presented a calculation of the spectral function of a smooth edge using a bosonization technique inspired by previous calculations for the uniform electron gas [6 8]. The theory contained a (physically motivated) cutoff on the number of hydrodynamic modes contributing to the spectral function for a given edge width $d$, and predicted a tunneling exponent increasing linearly with $d$. Similar results have been obtained by Han and Thouless [9, 10] using a Green's function method at finite temperature.

In this paper we refine our calculation in such a way that short wavelength modes are automatically excluded from contributing to the spectral function. Thus, the present theory is parameter free. We also extend our calculations to finite temperature 11] and obtain the low voltage/temperature scaling of the spectral function and of the tunneling current. Our numerical results, in the sharp edge limit, are found to be in good agreement with a recent experiment by Chang et al. [12].

Let us begin by writing down the microscopic Hamiltonian within the LLL in terms of density fluctuations relative to the equilibrium density profile $\rho_{0}(y)$ :

$$
H=\frac{1}{2} \int_{\text {edge }} \frac{e^{2}}{\left|\vec{r}-\vec{r}^{\prime}\right|} \delta \rho(\vec{r}) \delta \rho\left(\vec{r}^{\prime}\right) d^{2} r d^{2} r^{\prime}
$$

where $\rho(\vec{r})=\delta \rho(\vec{r})+\rho_{0}(y)$ is the density operator projected in the LLL. The hamiltonian (11) is diagonal in terms of the operators [5]

$$
b_{n k}=\frac{1}{\sqrt{k l^{2} \bar{\rho} L}} \int_{0}^{L} d x e^{-i k x} \int_{0}^{d} d y f_{n k}(y) \delta \rho(x, y),
$$

where $f_{n k}(y)$ are the solutions of the equation

$$
\int_{0}^{d} K_{0}\left(k\left|y-y^{\prime}\right|\right) f_{n k}\left(y^{\prime}\right) \frac{\rho_{0}^{\prime}\left(y^{\prime}\right)}{\bar{\rho}} d y^{\prime}=\frac{1}{\lambda_{n k}} f_{n k}(y),
$$

and $K_{0}(y)$ is the modified Bessel function. They form a complete set and satisfy the orthonormality condition $\int_{0}^{d} f_{n k}(y) f_{m k}(y) \frac{\rho_{0}^{\prime}(y)}{\bar{\rho}} d y=\delta_{n m}$, and vanish outside the interval $[0, d]$. Equation (3) is equivalent to the eigenvalue problem obtained by Aleiner and Glazman 13 from their hydrodynamical treatment of the collective modes of the edge. The eigenfunction of the $n$-th branch has $n$ nodes in the $y$ direction and energy $\omega_{n k}=k \bar{\nu} e^{2} / \lambda_{n k} \pi$, where $\bar{\nu}=2 \pi l^{2} \bar{\rho}$ is the usual filling factor in the bulk. It has been shown in Ref. 河 that the operators $b_{n k}$ satisfy boson commutation relations in the long wavelength limit $(k l \ll 1$ and $n \ll d / l)$.

The electronic spectral function can be obtained within the independent boson model (IBM) [6.14], which describes a single localized electron electrostatically coupled to density fluctuations. The IBM hamiltonian is

$$
\begin{aligned}
H_{I B M} & =\sum_{n k>0} \hbar \omega_{n k} b_{n k}^{\dagger} b_{n k}+ \\
& +\psi^{\dagger}(\vec{r}) \psi(\vec{r}) \sum_{n k>0} M_{n k}(y)\left[b_{n k}^{\dagger} e^{i k x}+b_{n k} e^{-i k x}\right],
\end{aligned}
$$

where $\psi^{\dagger}(\vec{r})$ is the field operator that creates an electron in the LLL coherent state (gaussian) orbital centered at $\vec{r}$, which is coupled to the bosons by the matrix element

$$
M_{n k}(y)=\frac{2 e^{2}}{\lambda_{n k}} \int d y^{\prime} e^{-\left(y-y^{\prime}\right) / l^{2}} e^{-k^{2} l^{2} / 4} f_{n k}\left(y^{\prime}\right) \sqrt{\frac{k \bar{\rho}}{\pi L}} .
$$

The form of the matrix elements differs from the one used in Ref. [5] by the presence of the gaussian convolution. The main consequence is that $M_{n k}(y)$ is found 
to vanish exponentially for large $n$ and/or $k$, and therefore short wavelength modes are automatically excluded. The hamiltonian (4) can be solved by standard methods [14, within the one-electron Hilbert space. The fermionic Green's function $G_{>}(y ; t)=-i\left\langle\psi(\vec{r}, t) \psi^{\dagger}(\vec{r}, 0)\right\rangle$ is proportional to

$$
\begin{gathered}
\exp \left\{\sum _ { n k > 0 } \frac { M _ { n k } ^ { 2 } ( y ) } { \omega _ { n k } ^ { 2 } } \left[\left(N_{n k}+1\right)\left(e^{-i \omega_{n k} t}-1\right)\right.\right. \\
\left.\left.+N_{n k}\left(e^{i \omega_{n k} t}-1\right)\right]\right\},
\end{gathered}
$$

where $N_{n k}$ is the Bose occupation factor. The local density of states is given by the spectral function $A_{>}(y, \omega)$, which is defined as the Fourier transform of $i G_{>}(y, t) / 2 \pi$. From eq. (6) it can be easily shown 15] that $A_{>}(y, \omega)$ satisfies the integral equation

$$
\omega A_{>}(y, \omega)=\int_{-\infty}^{\infty} \frac{g_{y}(\Omega)}{1-e^{-\Omega / k_{B} T}} A_{>}(y, \omega-\Omega) d \Omega,
$$

where

$$
g_{y}(\Omega)=\sum_{n k} \frac{M_{n k}(y)^{2}}{\omega_{n k}}\left[\delta\left(\Omega-\omega_{n k}\right)+\delta\left(\Omega+\omega_{n k}\right)\right] .
$$

In general, $g_{y}(\Omega)$ can be obtained from the imaginary part of the density-density response function $\chi\left(\vec{r}, \vec{r}^{\prime}, \Omega\right)$,

$$
g_{y}(\Omega)=-\int d^{2} r^{\prime} d^{2} r^{\prime \prime} v\left(\vec{r}-\vec{r}^{\prime}\right) v\left(\vec{r}-\vec{r}^{\prime \prime}\right) \frac{\operatorname{Im} \chi\left(\vec{r}^{\prime}, \vec{r}^{\prime \prime}, \Omega\right)}{\Omega \pi},
$$

where $v(r)$ has the Fourier transform $v(k)=$ $\left(2 \pi e^{2} / k\right) \exp \left(-(k l)^{2} / 4\right)$. This important connection betweeen the spectral function and the dynamical structure factor appears to be a general property of quasiclassical collective states in the LLL. In the case of a uniform electron liquid in the LLL equation (9) was first written down by Haussmann [7].

If the function $g_{y}(\Omega)$ has a finite limit for $\Omega \rightarrow 0$, at sufficiently small $\omega$ and $T\left(\omega, T \ll \omega_{0}\right)$ the spectral function obeys the scaling relation

$$
A_{>}(y, \omega) \propto T^{g_{y}(0)-1} f\left(\frac{\omega}{k_{B} T}, g_{y}(0)\right)
$$

where [10] $f(x, \alpha)$ has a finite limit for $x \rightarrow 0$ and behaves as $x^{\alpha-1}$ for $x \gg 1$; in particular $f(x, 2)=x /\left(1-e^{-x}\right)$.

The tunneling current $I(V)$ for vertical tunneling experiments between a smooth edge and a metallic contact satisfies a similar scaling relation [16],

$$
I(V) \propto T^{g_{y}(0)} h\left(\frac{V}{k_{B} T}, g_{y}(0)\right),
$$

where

$$
h(x, \alpha)=\int f(y, \alpha) \frac{e^{-x}-1}{e^{y-x}+1} d y
$$

is linear at small $x$ and proportional to $x^{\alpha}$ at large $x$. Therefore Ohm's law will be satisfied for $V \ll k_{B} T$, while for $V$ larger than $k_{B} T$ but still small on the electronic energy scale $\omega_{0}$ one gets a power law $I(V) \sim V^{g_{y}(0)}$. The normal Fermi-liquid behaviour is recovered for $g_{y}(0)=1$ $(h(x, 1)=x$ at any $x)$.

The calculation of the exponent $g_{y}(0)$ is easily performed. Neglecting the weak nonlinearity of the $n=0$ mode we obtain $\alpha=g_{y}(0) \simeq \sum_{n} \beta_{n}(y)$, where

$$
\beta_{n}(y)=\frac{1}{\bar{\nu}} f_{n 0}^{2}(y) e^{-n^{2} / 2 m^{2}}
$$

and $m=d \sqrt{2} / \ell \pi$. The gaussian factor accounts for the convolution with the gaussian wavefunction in equation (5).

We observe that independently of the shape of the density profile $\beta_{0}(y)=1 / \bar{\nu}$, with negligible corrections arising from the weak nonlinearity of the dispersion of the $n=0$ mode. In the sharp edge limit, when only one branch of edge waves exists, we obtain $A_{>}(\omega) \sim \omega^{1 / \bar{\nu}-1}$. For $\bar{\nu}=1 / 3$ this agrees with both CLL theory and experiment [2]. The exponent $g_{y}(0) \simeq d / l \bar{\nu}$ increases linearly with $d$ and therefore in the limit $d \rightarrow \infty$ (limit of infinitely smooth edge) the tunneling density of states vanishes at low energy faster than any power law. This is consistent with previous results for the uniform system [6, 7 .

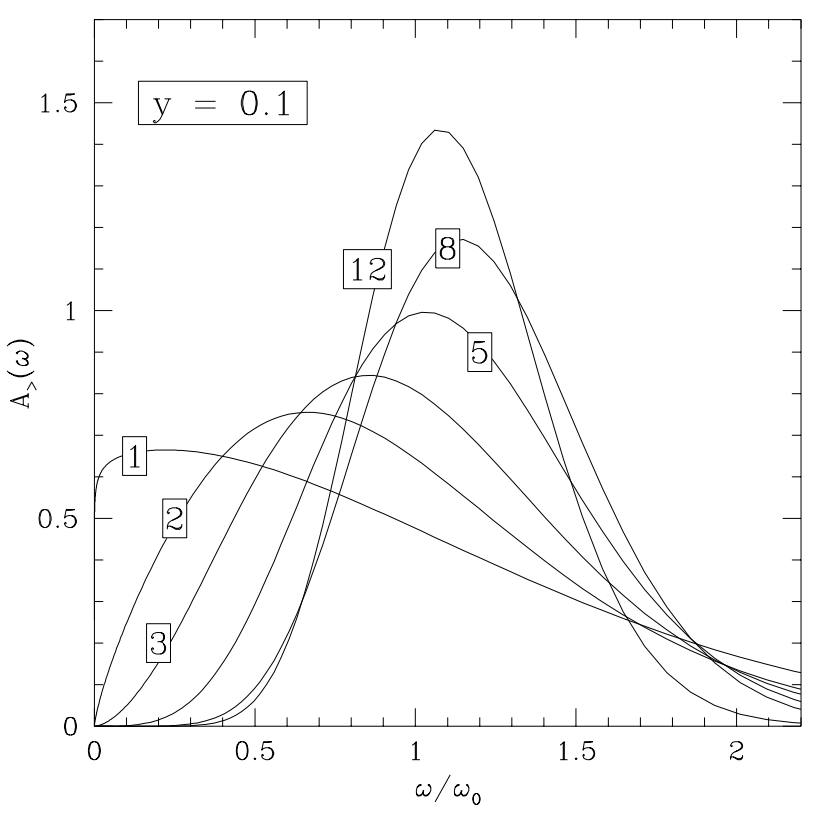

FIG. 1. Electronic spectral function $A_{>}(\omega)$ as a function of $\omega / \omega_{0}$, where $\omega_{0}=\bar{\nu} e^{2} / \pi l$, for edges of a $\bar{\nu}=1 \mathrm{QH}$ system with $m=1,2,3,4,6$ and 12, at an intermediate position $y=0.6 d$. Logarithmic corrections to the edge magnetoplasmon dispersion are here neglected. 


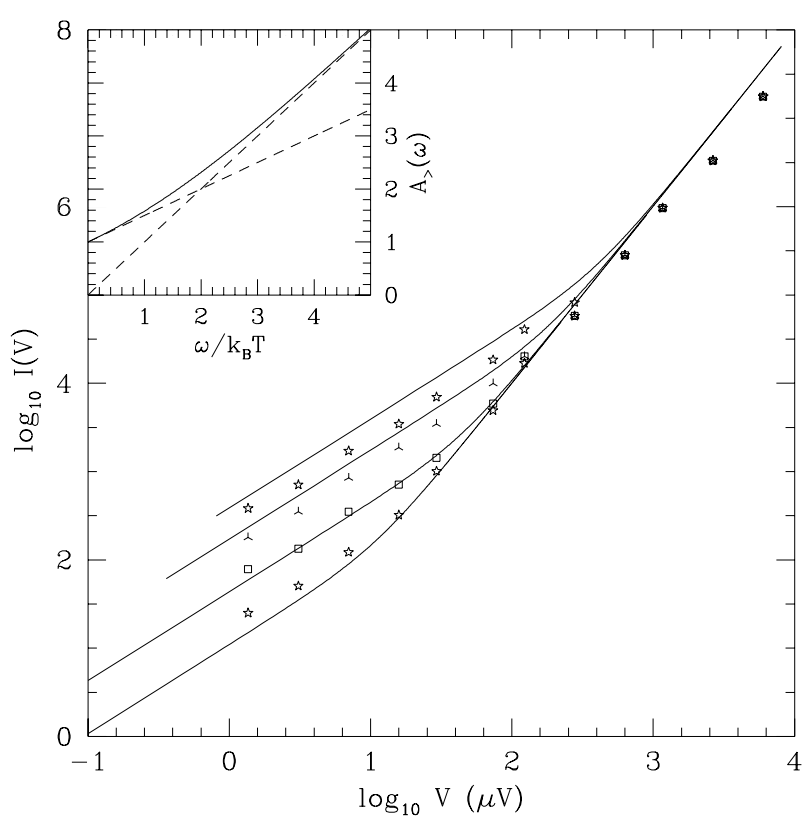

FIG. 2. Tunneling current $I(V)$ between a metallic contact and a sharp edge of a $\nu=1 / 2 \mathrm{QH}$ fluid at various temperatures, on a bilogarithmic scale. Full curves give the present result, points are the experimental data by Chang et al. 12. The inset shows the corresponding small- $\omega$ spectral function $A_{>}(\omega)$, on a linear scale, compared with the low and high frequency asymptotic behaviours (dashed lines).

In Figure 11 we present our numerical results for the full electronic spectral function, calculated from eq. (7) for different edge widths $d$. Notice that the calculation is free of adjustable parameters. The eigenfunctions $f_{n k}(y)$ and eigenfrequencies $\omega_{n k}$ have been calculated solving numerically equation (3) at finite $k$ in the special case $\rho_{0}(y)=\bar{\rho}\left[\frac{1}{\pi}\right.$ asn $\left.\frac{2 y-d}{d}+\frac{1}{2}\right]$ [17]. This form of $\rho_{0}(y)$ allows an analytical solution at small $k$, which gives $\beta_{n}(y)=\frac{1}{\bar{\nu}} T_{n}^{2}\left(\frac{2 y-d}{d}\right)\left(2-\delta_{n 0}\right) e^{-n^{2} / 2 m^{2}} \sim e^{-n^{2} / 2 m^{2}} / \bar{\nu}$, where $T_{n}(y)$ is the $n$-th Chebyschev polynomial.

Figure 2 compares our low-frequency results with the experimental data by Chang et al. 12 for tunneling between a metallic contact and a nominally atomically sharp edge of a $\nu=1 / 2 \mathrm{QH}$ system. The current has been obtained from equations (11-12) assuming that only the $n=0$ charged mode contributes to the response, i.e. with an exponent $\alpha=2$. Both the Ohmic behaviour at low voltages and the non-Fermi liquid behaviour at larger voltages are in good agreement with experiment. In contrast to this, a recent composite fermion theory by Shytov et al. 18 predicts $\alpha=3$ in this case.

In conclusion we have obtained via a bosonization approach a general relation between the one-electron spectral function and the dynamical structure factor. This allows a parameter-free calculation of the spectral function which interpolates between the sharp edge limit and the uniform electron gas and is in good agreement with experiments on sharp edges both at compressible and in- compressible filling factors.

We gratefully acknowledge support from NSF grant No. DMR-9706788 and from INFM. We also acknowledge the hospitality of the Max Planck Institute for Physics of Complex Systems in Dresden where part of the work has been done.

[1] X. G. Wen, Phys. Rev. B 41, 12838 (1990); Phys. Rev. B 44, 5708 (1991); Int. Journ. Mod. Phys. B 6, 1711 (1992).

[2] A. M. Chang, L. N. Pfeiffer, and K. W. West, Phys. Rev. Lett. 77, 2538 (1996).

[3] C. de Chamon and X. G. Wen, Phys. Rev. B 49, 8227 (1994).

[4] A. M. Chang, Solid State Comm. 74, 871 (1990); C. W. L. Beenakker, Phys. Rev. Lett. 64, 216 (1990); D. B. Chklovskii, B. I. Shklovskii, and L. I. Glazman, Phys. Rev. B 46, 4026 (1992).

[5] S. Conti and G. Vignale, Phys. Rev. B 54, 14309 (1996).

[6] P. Johansson and J. M. Kinaret, Phys. Rev. B 50, 4671 (1994).

[7] R. Haussmann, Phys. Rev. B 53, 7357 (1996).

[8] I. L. Aleiner, H. U. Baranger, and L. I. Glazman, Phys. Rev. Lett. 74, 3435 (1995); I. L. Aleiner and L. I. Glazman, Phys. Rev. B 52, 11296 (1995).

[9] J. H. Han and D. J. Thouless, Phys. Rev. B 55, 1926 (1997).

[10] J. H. Han, preprint cond-mat/9702074 (1997).

[11] Finite-temperature results were first presented by J. H. Han in Ref. 10.

[12] A. M. Chang, L. N. Pfeiffer, and K. W. West, preprint (1997).

[13] I. L. Aleiner and L. I. Glazman, Phys. Rev. Lett. 72, 2935 (1994).

[14] G. D. Mahan, Many-particle Physics (Plenum Press, New York, 1990).

[15] P. Minnhagen, Phys. Lett. 56A, 327 (1976).

[16] For sharp edges this scaling has been obtained by C. L. Kane and M. P. A. Fisher [Phys. Rev. Lett. 68, 1220 (1992)].

[17] This form is essentially equivalent to the one chosen in 13. for a gate-confined system.

[18] A. V. Shytov, L. S. Levitov, and B. I. Halperin, preprint cond-mat/9703246 (1997). 